\title{
Comparison of Ultrafast Tracking Anesthesia versus Conventional Strategy in Postoperative Management of Off-Pump Coronary Artery Bypass Surgery
}

\author{
Amarja S. Nagre ${ }^{1}$ \\ ${ }^{1}$ Department of Cardiac Anaesthesia, MGM Medical College and \\ MCRI, Aurangabad, Maharashtra, India
}

J Card Crit Care TSS 2018;2:32-35

\begin{abstract}
Address for correspondence Amarja Nagre, DM, FCA, FICCC, C-3, Assistant Professor, Department of Cardiac Anaesthesia, MGM Medical College and MCRI, Row House, Muthiyan Residency, Deepnagar, Aurangabad, Maharashtra 431001, India (e-mail: dramarja.1@gmail.com).
\end{abstract}

\begin{abstract}
Keywords

- ultrafast tracking

- postoperative ventilation

- off-pump coronary artery bypass
\end{abstract}

Introduction Conventional strategy in the postoperative ventilation was a long-established practice in former times but now fast tracking is an acknowledged technique. This study was embarked to assess the impact of ultrafast tracking on patient recovery and length of stay in intensive care unit (ICU) compared with conventional methods in off-pump coronary artery bypass (OPCAB) patients.

Methods Fifty patients were enrolled in each group. Exclusion criteria were patients with ejection fraction $<30 \%$, hemodynamically unstable, on intra-aortic balloon pump, associated valvular heart disease, and intraoperative conversion to on-pump coronary artery bypass grafting. Ultrafast tracking of anesthesia (UFTA) protocol included induction with low dose fentanyl, propofol, and vecuronium. Maintenance of anesthesia was done with sevoflurane, fentanyl, and atracurium whereas analgesia was done with tramadol and paracetamol.

Results Statistical analysis was done with SPSS 21.0 program. Fischer's test, chi-square test, independent $t$-test/Mann-Whitney $U$ tests were used for calculations. Extubation time was statistically significant $(p=0.0001)$. Shift to ward was $3.96 \pm 0.73$ days in UFTA group and $4.34 \pm 0.66$ days in conventional group which proved statistically significant $(p=0.0073)$. No patients in both groups had postoperative myocardial infarction, stroke, low cardiac output, or mediastinitis. Renal failure and mortality were $2 \%$ in conventional group. Hypothermia, pacemaker dependency, blood transfusion, inotrope score, atrial fibrillation, re-exploration, and reintubation were monitored. No reintubation occurred in UFTA group.

Conclusion UFTA appears to be safe and effective in OPCAB patients with early shift from ICU without any major complications.

\section{Introduction}

Interest in fast tracking in cardiac anesthesia has been rekindled as the pressure on health system is increasing by virtue of growth in patient number, patients with various comorbidities, and expensive resources. ${ }^{1}$ Off-pump coronary artery bypass (OPCAB) has shown outstanding outcomes with the benefit of less usage of resources, reduced blood loss, decreased morbidity, and shortened length of hospital stay. ${ }^{2}$ Limiting the costs while maintaining quality of healthcare has become an imperative concept. ${ }^{3}$

The undisputed conventional ventilation strategy has now been replaced by fast tracking of anesthesia (FTA). It becomes practicable by implementation of multidisciplinary patient management strategies. ${ }^{4} \mathrm{~A}$ decreased rate of postoperative low cardiac output syndrome and mortality is seen in the

\section{License terms}

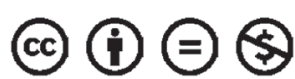


fast track group. ${ }^{5}$ FTA is cost-effective without increase in complications and this has led to the universal adoption of this protocol. ${ }^{3}$

The pre-eminent strategy of ultrafast tracking of anesthesia (UFTA) is abbreviating the prolonged ventilatory support, minimizing the intensive care unit (ICU) stay, and promoting early discharge from hospital. ${ }^{6}$ The essence of UFTA is titration of short acting anesthetic drugs, maintenance of body temperature, and early extubation with effective analgesia. ${ }^{7}$ However, UFTA with extubation in the operating room can be performed only in selected group of patients. ${ }^{8}$

This study was undertaken to analyze the factors associated with ultrafast tracking in OPCAB patients and to assess its impact on patient recovery and ICU stay as compared with conventional methods.

\section{Methods}

Fifty patients were enrolled in the study who underwent OPCAB surgery. Demographics and operation-related data are mentioned in - Table 1. Inclusion criteria were patients posted for OPCAB surgery including left main stenosis. Exclusion criteria were hemodynamically unstable patients, patients on intra-aortic balloon pump (IABP), with renal dysfunction, ejection fraction $<30 \%$, associated valvular heart disease, acute myocardial infarction (MI), on temporary pacemaker, and those patients who underwent intraoperative conversion to on-pump coronary artery bypass grafting. Ultrafast track protocol was followed in all patients. Oral lorazepam $2 \mathrm{mg}$ the previous night and oral metoprolol $12.5 \mathrm{mg}$ in the morning were administered. In the operating room, standard American Society of Anesthesiologists monitoring was applied and right internal jugular vein cannulation and femoral arterial line insertion were done. Induction was done intravenously with fentanyl $4 \mu \mathrm{g} / \mathrm{kg}$, midazolam $0.04 \mathrm{mg} / \mathrm{kg}$, propofol 0.5 to $1 \mathrm{mg} / \mathrm{kg}$, rocuronium $0.6 \mathrm{mg} / \mathrm{kg}$ and maintenance of anesthesia with fentanyl, propofol, atracurium intermittently, and sevoflurane. Last dose of muscle relaxant was given at the time of sternal wiring. Local anesthetic infiltration with bupivacaine $0.25 \%$ injection was done at skin closure. ${ }^{9}$ Body temperature was maintained within normal limits. Tramadol $100 \mathrm{mg}$ injection was given during closure. Paracetamol 20 $\mathrm{mg} / \mathrm{kg}$ and tramadol $50 \mathrm{mg}$ intravenous were given postoperatively every 6 hours.

Ultrafast tracking protocol in ICU was as follows-after shifting the patient to ICU, arterial blood gas (ABG) analysis was done and repeated on continuous positive airway pressure mode of ventilation and then 20 minutes after extubation. Reversal of muscle relaxants was done and patient extubated after vigilant monitoring of vital signs by electrocardiography, invasive blood pressure, pulse oximetry, central venous pressure, blood collection in drains, urine output, and chest X-ray. Pain intensity after extubation was judged by numeric pain scale (0-no pain to $10-$ maximum pain) every hour.

Table 1 Operation-related data

\begin{tabular}{|c|c|c|c|}
\hline Demographics and operation-related data & UFTA group & Conventional group & $p$-Value \\
\hline Age $(y)$ & $58.62 \pm 9.92$ & $57.88 \pm 8.63$ & 0.6917 NS \\
\hline \multicolumn{4}{|l|}{ Sex } \\
\hline Male & $15(30 \%)$ & $13(26 \%)$ & \multirow[t]{2}{*}{$0.6725 \mathrm{NS}$} \\
\hline Female & $35(70 \%)$ & $37(74 \%)$ & \\
\hline Ejection fraction (\%) & $50.42 \pm 10.33$ & $48.82 \pm 7.65$ & 0.3809 NS \\
\hline Ward shift day & $3.96 \pm 0.73$ & $4.34 \pm 0.66$ & $0.0073 \mathrm{~S}$ \\
\hline Diabetes mellitus & $6(12 \%)$ & $4(8 \%)$ & 0.5028 NS \\
\hline Hypertension & $6(12 \%)$ & $6(12 \%)$ & $1.000 \mathrm{NS}$ \\
\hline Preop MI & $9(18 \%)$ & $10(20 \%)$ & 0.8025 NS \\
\hline Preop renal efficiency & $0(0 \%)$ & $2(4 \%)$ & 0.4949 NS \\
\hline Preop chronic obstructive lung disease & $2(4 \%)$ & $2(4 \%)$ & $1.000 \mathrm{NS}$ \\
\hline Preop peripheral vascular disease & $1(2 \%)$ & $0(0 \%)$ & $1.000 \mathrm{NS}$ \\
\hline Preop neurological deficit & $0(0 \%)$ & $0(0 \%)$ & $1.000 \mathrm{NS}$ \\
\hline Time to extubation in hours (hours) & $2[1-3.3]$ & $5.775[4.45-7.15]$ & $0.0001 \mathrm{~S}$ \\
\hline Category & UFTA group & Conventional group & $p$-value \\
\hline Postop MI (\%) & 0 & 0 & $1.000 \mathrm{NS}$ \\
\hline Postop low cardiac output (\%) & 0 & 0 & $1.000 \mathrm{NS}$ \\
\hline Postop renal failure (\%) & 0 & $1(2 \%)$ & $1.000 \mathrm{NS}$ \\
\hline Postop stroke (\%) & 0 & 0 & $1.000 \mathrm{NS}$ \\
\hline Postop mediastinitis (\%) & 0 & 0 & $1.000 \mathrm{NS}$ \\
\hline Postop mortality (\%) & 0 & $1(2 \%)$ & $1.000 \mathrm{NS}$ \\
\hline
\end{tabular}

Abbreviations: MI, myocardial infarction; NS, not significant; S, significant; UFTA, ultrafast tracking of anesthesia. 


\section{Results}

The data were transferred to the SPSS 21.0 (IBM Inc.) program for statistical analysis. Fischer's test, chi-square test, and independent $t$-test/Mann-Whitney $U$ test were used to calculate statistical significances. $p$-values $<0.05$ are considered statistically significant. Demographic data of preoperative patient variables were comparable in both groups. Extubation time was statistically significant $(p=0.0001)(-$ Fig. 1$)$. Shift to ward was $3.96 \pm 0.73$ days in UFTA group and $4.34 \pm 0.66$ days in conventional group, which was statistically significant $(p=0.0073)$ ( - Fig. 2) as shown in $\boldsymbol{- T a b l e ~ 1 . ~ N o ~ p a t i e n t s ~ i n ~}$ both groups had postoperative MI, stroke, low cardiac output, and mediastinitis. Renal failure and mortality were $2 \%$ in conventional group. Hypothermia, pacemaker dependency, blood transfusion, inotrope score, atrial fibrillation (AF), re-exploration, and reintubation were monitored. No reintubation and readmission to ICU occurred in UFTA group.

\section{Discussion}

We focused on ultrafast tracking interventions particularly designed for early extubation within 3 hours of completion of surgery like low-dose opioid-based general anesthesia and use of time-directed extubation protocol. ${ }^{10}$ Absolute care was executed to avoid the postoperative noncardiac morbidity defined as intubation period $>24$ hours, reintubation, postoperative renal failure, sternal infection, and sepsis. ${ }^{1}$ Fast tracking may precipitate premature extubation

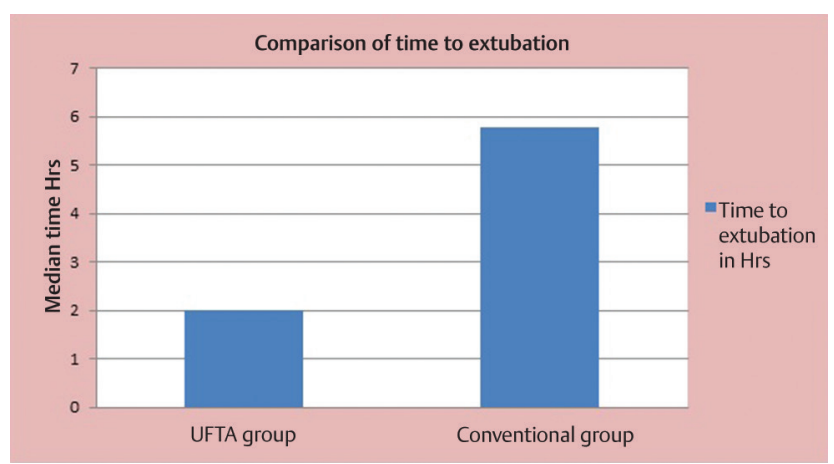

Fig. 1 Comparison of time to extubation. UFTA, ultrafast tracking of anesthesia.

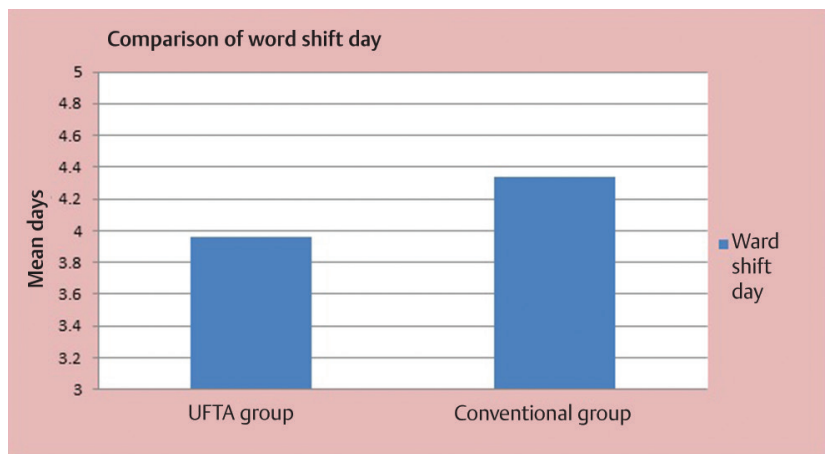

Fig. 2 Comparison of ward shift day. UFTA, ultrafast tracking of anesthesia. thus increasing the risk of reintubation, however, without increase in morbidity or mortality if low dose opioid regimen is used. ${ }^{11}$ Early extubation may increase respiratory and cardiac workload causing myocardial ischemia, hypothermia, shivering, reintubation, inadequate analgesia, and mortality. Mediastinal bleeding requiring re-exploration is a potential problem in cardiac surgery wherein a secured airway is desired. The main reasons for delayed fast track extubation are excess bleeding resulting in re-exploration, high inotropes, deep sedation, altered sensorium, and hemodynamically unstable patient. ${ }^{12}$

However, benefits of early extubation in cardiac surgery include decreased laryngotracheal trauma, reduction in stress associated with weaning from ventilation, less requirement of sedatives, and avoidance of ventilator disposables. Moreover, patients are transferred to lower dependency unit earlier, and fewer nursing staff are requested to manage each patient. Ultimately, the cost saving is ascertained. ${ }^{13}$

Early extubation further decreases the usage of ventilator, oxygen, ABG sampling, suction catheters, gloves, power consumption, manpower consumption. This ultimately minimizes the cost analogous to cardiac surgery care. ${ }^{14}$ It improves resource utilization compared with late extubation..$^{15}$ The undesirable effects of prolonged ventilation such as accidental extubation, lung infections, tracheal suction causing pulmonary hypertensive crisis, mucus plugging, and atelectasis are alleviated. Furthermore, early extubation allows patient to do respiratory physiotherapy earlier to reduce the complications like atelectasis and pneumonia. Improvement in diastolic compliance and overall cardiac performance are also the potential benefits. ${ }^{16}$ Extubation to spontaneous breathing is associated with decrease in intrapleural pressure and increase in stroke work and cardiac output. The ventricular function is also improved as the preload increases due to shifting of the capacitance blood volume toward the chest. ${ }^{17}$

In our study, the primary outcome was the risk of mortality. Secondary outcomes included postoperative complications, reintubation within 24 hours of surgery, time to extubation, and ICU length of stay. Hypothermia, pacemaker dependency, blood transfusion, high inotropes, AF, and re-exploration were also closely watched. Adequate analgesia was administered as it benefits by improving patient's participation in physiotherapy and early ambulation. ${ }^{18}$ We had significant results in terms of the parameters "time to extubation" and "shift to ward." Our exclusion criteria were stringent as previous published data have certain specifications as patient on IABP, inotropes, excessive bleeding, and atrial arrhythmias is associated with delayed extubation. Besides, the predictors for failure of immediate extubation plan were previous renal failure, cardiac reoperation, and preoperative IABP placement. ${ }^{15}$ The tracheal extubation criteria, ICU discharge criteria, and hospital discharge criteria were preset and followed scrupulously.

The extubation criteria were a cooperative alert patient with peripheral capillary oxygen saturation > 98\% with fraction of inspired oxygen $<60 \%$, end-tidal carbon dioxide $<45 \mathrm{~mm} \mathrm{Hg}$, and stable hemodynamics with minimal inotropes, no hypothermia or arrhythmias, and a normal ABG. 
Intensive care unit discharge criteria were hemodynamic stability, adequate urine output, no arrythmias or inotropes, no pacemaker dependency with all invasive lines removed, and having adequate pain control.

Hospital discharge criteria were full mobilization and tolerance of full diet.

Extubation within 1 to 6 hours do not increase perioperative cardiac or respiratory morbidity. The predominant issues are bleeding, respiratory insufficiency, and hypothermia. Thermoregulation is impaired perioperatively. Despite adequate rewarming, core temperature has an "afterdrop" due to temperature gradients between the core and the periphery. Moreover, shivering brings about an increase in metabolic rate and thereby the potential for myocardial ischemia. Hypothermia increases morbidity, so the concept of immediate extubation is viable only when the core body temperature is maintained. ${ }^{8}$ Monitoring the depth of anesthesia by bispectral index (BIS) subserves judicious use of sedatives while obviating intraoperative awareness. ${ }^{12}$ Thence, the anesthesia management and postoperative care determine extubation time and ultrafast tracking. ${ }^{19}$

\section{Limitations of study}

We did not monitor the depth of anesthesia by BIS. Early hospital discharge was not emphasized in our setup, but it should be encouraged in UFTA patients.

\section{Conclusion}

Ultrafast tracking of anesthesia certainly allays the complications akin to mechanical ventilation and thereby abbreviates the ICU stay. Thence, it is safe and efficacious in OPCAB patients. Optimization of analgesia and avoidance of hypothermia are the fundamentals in the safe conduct of UFTA. Ultimately, the resulting accelerated postoperative recovery contributes majorly to the results of cardiac surgery.

\section{Conflict of Interest}

None.

\section{Acknowledgment}

Special thanks to the Department of Cardiac Surgery for their support in execution of UFTA in OPCAB patients.

\section{References}

1 Kiessling AH, Huneke P, Reyher C, Bingold T, Zierer A, Moritz A. Risk factor analysis for fast track protocol failure. J Cardiothorac Surg 2013;8:47

2 Sellke FW, DiMaio JM, Caplan LR, et al; American Heart Association. Comparing on-pump and off-pump coronary artery bypass grafting: numerous studies but few conclusions: a scientific statement from the American Heart Association council on cardiovascular surgery and anesthesia in collaboration with the interdisciplinary working group on quality of care and outcomes research. Circulation 2005;111(21):2858-2864

3 Lawrence EJ, Nguyen K, Morris SA, et al. Economic and safety implications of introducing fast tracking in congenital heart surgery. Circ Cardiovasc Qual Outcomes 2013;6(2):201-207

4 Lezzi F, Summa M, Sarto PD, Munene J. Fast-track extubation in pediatric cardiothoracic surgery in developing countries. J Card Crit Care TSS 2017;1:18-20

5 Ender J, Borger MA, Scholz M, et al. Cardiac surgery fast-track treatment in a postanesthetic care unit: six-month results of the Leipzig fast-track concept. Anesthesiology 2008;109(1):61-66

6 Nagre AS, Jambures NP. Comparison of immediate extubation versus ultrafast tracking strategy in the management of off-pump coronary artery bypass surgery. Ann Card Anaesth 2018;21(2):129-133

7 Kandasamy A, Ramalingam SK, Simon HA, Arumugham S, Reddy BD, Krupananda H. Ultra fast-tracking versus a conventional strategy in valve replacement surgery. Indian J Anaesth 2013;57(3):298-300

8 Hemmerling TM, Romano G, Terrasini N, Noiseux N. Anesthesia for off-pump coronary artery bypass surgery. Ann Card Anaesth 2013;16(1):28-39

9 Hemmerling TM, Prieto I, Choinière JL, Basile F, Fortier JD. Ultra-fast-track anesthesia in off-pump coronary artery bypass grafting: a prospective audit comparing opioid-based anesthesia vs thoracic epidural-based anesthesia. Can J Anaesth 2004;51(2):163-168

10 Zhu F, Lee A, Chee YE. Fast-track cardiac care for adult cardiac surgical patients. Anesthesiology 2003;99:982-987

11 Myles PS, Daly DJ, Djaiani G, Lee A, Cheng DC. A systematic review of the safety and effectiveness of fast-track cardiac anesthesia. Anesthesiology 2003;99(4):982-987

12 Akhtar MI, Hamid M. Success and failure of fast track extubation in cardiac surgery patients of tertiary care hospital: one year audit. J Pak Med Assoc 2009;59(3):154-156

13 Gangopadhyay S, Acharjee A, Nayak SK, Dawn S, Piplai G, Gupta K. Immediate extubation versus standard postoperative ventilation: our experience in on pump open heart surgery. Indian J Anaesth 2010;54(6):525-530

14 Bainbridge D, Cheng DC. Early extubation and fast-track management of off-pump cardiac patients in the intensive care unit. Semin Cardiothorac Vasc Anesth 2015;19(2):163-168

15 Cheng DC, Karski J, Peniston C, et al. Early tracheal extubation after coronary artery bypass graft surgery reduces costs and improves resource use. A prospective, randomized, controlled trial. Anesthesiology 1996;85(6):1300-1310

16 Abdelwahab HH. Feasibility of ultrafast anaesthesia the first descriptive prospective study in Egypt. Egyptian J Cardiothorac Anesth 2011;3:643-651

17 Gall SA Jr, Olsen CO, Reves JG, et al. Beneficial effects of endotracheal extubation on ventricular performance. Implications for early extubation after cardiac operations. J Thorac Cardiovasc Surg 1988;95(5):819-827

18 Chakravarthy MR, Prabhakumar D. Anaesthesia for off-pump coronary artery bypass grafting -the current concepts. Indian J Anaesth 2007;51(4):334-343

19 Cheng DC. Regional analgesia and ultra-fast-track cardiac anesthesia. Can J Anaesth 2005;52(1):12-17 